\title{
A Method to Determine the Electrical Resistance of a Metallic Powder Mass under Compression
}

\author{
Juan Manuel Montes ${ }^{1}$, Francisco Gómez Cuevas ${ }^{2}$, Fátima Ternero ${ }^{1, *}$ (D), Raquel Astacio ${ }^{1}$, \\ Eduardo Sánchez Caballero ${ }^{1}$ and Jesús Cintas ${ }^{1}$ \\ 1 Metallurgy and Materials Engineering Group, Escuela Técnica Superior de Ingeniería, \\ Universidad de Sevilla, Camino de los Descubrimientos s/n, 41092 Sevilla, Spain; \\ jmontes@us.es (J.M.M.); rastacio@us.es (R.A.); esanchez3@us.es (E.S.C.); jcintas@us.es (J.C.) \\ 2 Department of Chemical Engineering, Physical Chemistry and Materials Science, \\ Escuela Técnica Superior de Ingeniería, Universidad de Huelva, Campus El Carmen, \\ Avda. Tres de Marzo s/n, 21007 Huelva, Spain; fgcuevas@dqcm.uhu.es \\ * Corresponding: fternero@us.es; Tel.: +34-95-448-7305
}

Received: 11 October 2017; Accepted: 3 November 2017; Published: 6 November 2017

\begin{abstract}
In this paper, a phenomenological model to predict the value of the electrical resistance of a compressed metal powder mass is proposed. The model, based on the experimental compressibility and resistivity-porosity curves, is useful in the field of the electrical resistance consolidation. In this area is often required to find out whether a certain mass of powder inside a die of specified inner section, and subjected to a certain compression, reaches a sufficiently small resistance value so that it can be consolidated by electrical means. The model also predicts the electrical resistance value of the powder mass in case of powders with no oxide layers, or after removing them mechanically or electrically by a previous activation process. The model predictions have been successfully validated through direct measurements of electrical resistance in powder aggregates both in as-received state and after electrical activation.
\end{abstract}

Keywords: electrical resistance; modeling; electrical sintering

\section{Introduction}

Nowadays, the electrical consolidation techniques are a competence to have in mind for the conventional route in powder metallurgy (PM) industrial processes. Grasso et al. [1] reviewed the different modalities of electrical sintering, generally known as field-assisted sintering techniques (FAST), all of them indeed very quick processes, although with some different underlying physical phenomena taking place. The main differences among these different modalities consist in the type of electric power source and the nature of the die (in particular whether it is electrically conductive or insulating). Associated with each technique arise problems that previously could have no interest.

In particular, for those techniques using electrically insulating dies, one of the aforementioned problems is to determine if a certain mass of powder, inside a die and subjected to a known uniaxial pressure, may or not be electrically consolidated. Often, the critical parameter is the value of the electrical resistance of the powder mass, which should not exceed a certain maximum value. Among these electrical consolidation techniques, in the so called Electrical Resistance Sintering (ERS), the power source is a transformer designed to supply a high intensity current, although with voltages usually under 10 V. In a similar way, for the technique known as Electrical Discharge Consolidation (EDC), the power source is usually a capacitors bank, charged with voltages of up to $200 \mathrm{~V}$. The reason for this maximum resistance value is the limited voltage that the power source can provide, $V_{\mathrm{M}}$, and the need of a minimum value of the current intensity, $I_{\mathrm{m}}$, which should pass through the powder mass to have an efficient consolidation process (in general, if this value is not surpassed the electronics of 
control stops the process). Nevertheless, the value of $I_{\mathrm{m}}$ imposed by the technical limitations of the equipment can increase because of the need of a higher value to ensure an adequate consolidation. This depends on the die geometry, the nature of the powder to consolidate and the applied pressure. The final value of $I_{\mathrm{m}}$ has to be known by trial and error or through the use of a process simulator. Once known $V_{\mathrm{M}}$ and $I_{\mathrm{m}}$, and according to the Ohm law, the powder mass to be consolidated should have an electrical resistance lower than $R_{\mathrm{M}}=V_{\mathrm{M}} / I_{\mathrm{m}}$. Thus, the particular values of $V_{\mathrm{M}}$ and $I_{\mathrm{m}}$ depend on the considered technique and its technical limitations, mainly due to the control and/or security electronics. For instance, in the case of the ERS technique, usually implemented through resistance welding machines, the values $V_{\mathrm{M}} \sim 10 \mathrm{~V}$ and $I_{\mathrm{m}} \sim 100$ A lead to a maximum resistance value making possible the process of $R_{\mathrm{M}}=0.1 \Omega$. In the case of the EDC technique, usually carried out with stud welding machines, the typical values are $V_{\mathrm{M}} \sim 200 \mathrm{~V}$ and $I_{\mathrm{m}} \sim 100 \mathrm{~A}$, leading to a maximum resistance value of $R_{\mathrm{M}}=2 \Omega$.

The electrical resistance of the powder mass logically depends on its porosity, and this decreases with increasing pressure. Therefore, the higher the pressure, the lesser is the porosity and therefore the lower is the electrical resistance. However, pressure not only helps to reduce the porosity [2,3]; also, due to the friction between particles, pressure forces the descaling of the dielectric layers (mainly oxides, but also hydrides and other chemical compounds) that normally cover the powder metallic particles [4]. Both phenomena lead to decreasing the effective resistivity of the powder mass. These oxides have a high influence on the apparent value of the electrical resistance, to the point that may have more influence than the own porosity. The problem has been widely studied [5-7]. The difficulty of the problem does not only resides in the fact of experimentally determining the thickness and the electrical resistivity of the oxide layers (generally dielectric) covering the micrometric powder particles used in powder metallurgy. The exact chemical state of these oxides is generally difficult to know, and hydroxides can appear together to the oxides. Even considering the only presence of oxides, metal atoms can appear with different oxidation states, increasing the complexity of the problem. This is not the case, for instance, for the presence of alumina $\left(\mathrm{Al}_{2} \mathrm{O}_{3}\right)$ in aluminum, but yes for iron, with the possible presence of $\mathrm{FeO}, \mathrm{Fe}_{3} \mathrm{O}_{4}, \mathrm{Fe}_{2} \mathrm{O}_{3}$ and $\mathrm{Fe}_{1-x} \mathrm{O}$ (wüstite, a non-stoichiometric phase). In this situation, the oxygen-richest phase will appear near the powder surface. Another sources of uncertainty are the crystalline or amorphous nature of the oxide layers, the number of vacancies and impurities in the oxide and any other factor altering the conductivity [8,9]. Thus, the nanometric thickness of the oxides diminishes the resistivity according to the Fuchs-Sondheimer law [10,11]. Fortunately, despite the aforementioned effects, the conductivity of the oxide layer does not differ in more than one order of magnitude with respect to the conductivity of the bulk oxide, which can be found in the literature.

Therefore, because of the possible presence of multiple species of iron oxides, the one with the higher resistivity, corresponding to $\mathrm{Fe}_{2} \mathrm{O}_{3}$, should be considered, with a value of $2.1 \mathrm{k} \Omega \cdot \mathrm{m}$ for a layer of $4.5 \mathrm{~nm}$ thick [10]. However, even with resistivity and thickness values perfectly determined, the problem would remain partially unsolved, because the oxide layers can be mechanically broken by the applied pressure, or a dielectric breakdown produced by the current passing.

Thus, the effect of increasing the pressure is double: reducing the porosity of the powder mass, and, on the other hand, helping to the descaling of the oxide layers, favoring metal-metal interparticle contacts, which drastically reduced the value of the effective resistivity of the material.

In order to avoid the effect of these oxide layers, electrical consolidation processes working with low voltage $(\sim 10 \mathrm{~V})$, can start with a very short activation stage. This stage applies high or medium voltage $(>200 \mathrm{~V})$ to force the dielectric breakdown of the dielectric layers. Thus, the powder mass achieves a lower electrical resistance, allowing to deal with the electrical consolidation of higher powder columns.

In this paper, we present a method to estimate the resistance value of a known powder mass under compression, as well as the value of this resistance if the mass constituent particles were free of oxides. This calculation method allows predicting whether a certain powder column, under a known 
pressure, could be successfully consolidated with an electrical technique, reducing the number of experiences to attain the appropriate conditions of the process.

\section{Compressibility and Resistivity-Porosity Curves}

The starting point of the method is the determination of two curves that characterize the powder under compression. The first is the compressibility curve of the powder, which collects information as to how the porosity (or relative density) of the powder mass varies when it is subjected to an increasing compression. The second curve records how the electrical resistivity of the powder mass varies (decreases) under an increasing compression. Preparing the proper experimental set-up, both curves can be simultaneously determined.

For the determination of both curves an insulating die is needed, i.e., an alumina tube with a steel hoop and the gap filled with molten lead. Once solidified, lead guarantees a perfect coupling, avoiding the die breakage. The alumina choice mainly obeys to its very high resistivity (approximately $10^{14} \Omega \cdot \mathrm{m}$ at room temperature), ensuring the absence of lateral leaks. The die is closed at the top and bottom by two copper ( $99.9 \%$ purity) punches/electrodes with the powder mass to be tested inside. The electrical resistance of the punches can be either determined by measurement with a micro-ohmmeter, or through the appropriate theoretical calculations in case of knowing the electrical properties of the material. Each punch is connected to two cables: the outer ones used to inject current, and the inner ones to measure the voltage drop. A PVC base is used to make possible the connection in the lower electrode without making necessary passing the cables through the steel hoop and the ceramic die. As the used micro-ohmmeter measures both the intensity provided and the voltage drop, it is capable to directly provide the value of the electrical resistance, to which will be necessary to discount the electrical resistance of the electrodes. A four probes micro-ohmmeter (Kelvin bridge [12]) is necessary to take advantage of this set-up, shown in Figure 1. In case of measurements under very low pressure conditions (with a porosity very close to the initial one), a kilo-ohmmeter would be necessary.

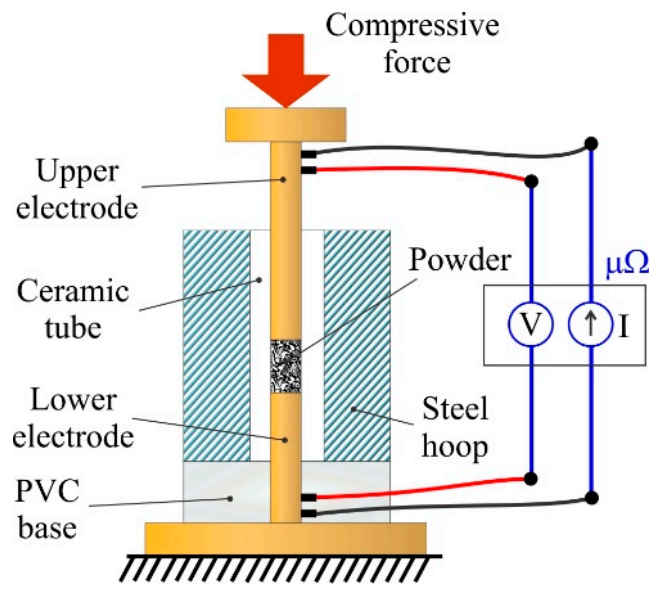

Figure 1. Experimental set-up for the determination of the compressibility and resistivity curves.

For the compression test, an instrumented Universal Testing Machine (Instron 5505, Norwood, MA, USA) can be used. The test begins placing together both electrodes without powder, to calibrate the zero displacement of the upper plate of the machine, once in contact with the upper electrode. Once registered, the die loaded with powder and closed by the upper and lower punches is placed on position. The powder is not just poured into the die to reach the apparent density of the powder [13], but it is also vibrated until reaching its tap porosity [14], a reproducible situation for all the experiences. Then, the upper plate is gently lowered until making contact with the upper punch, being registered the height of the powder column and measured the electrical resistance. The initially null applied 
force is then increased up to $20 \mathrm{~N}$ (although this value depends on the nature and characteristics of the metallic powder), and the height of the column and resistance are again registered, and so on. At the beginning is enough with only $20 \mathrm{~N}$ increments because important variations take place, subsequently these increments can be increased up to even $200 \mathrm{~N}$. Ideally, the register of the powder column height variations should be done with an extensometer, although the height of the powder column can also be determined by the displacement of the machine bench, as was done in this work. In this latter case, it must be considered that the machine frame is not ideally stiff, and its deformation must be taken into account (this can be determined by registering the displacements produced in a compression experience of electrode against electrode, without any powder).

From the actual powder column height, $H$, determined in each measure, the corresponding value of the porosity of the powder mass, $\Theta$, can be calculated using the expression:

$$
\Theta=1-\frac{M}{\gamma_{\mathrm{M}} \cdot S_{\mathrm{N}} \cdot H}
$$

where $M$ is the powder mass, $\gamma_{M}$ is the bulk material density and $S_{N}$ is the inner section of the die. The value of $H$ is computed as the difference between the registered position of the upper plate and the initially measured position of electrode against electrode.

On the other hand, knowing the measured electrical resistance in the ohmmeter, $R$, the corresponding electrical resistivity value can be determined by:

$$
\rho=R \cdot \frac{S_{\mathrm{N}}}{H}
$$

For each applied load, $F$, the nominal pressure, $P_{\mathrm{N}}$, is determined by:

$$
P_{\mathrm{N}}=F / S_{\mathrm{N}}
$$

Once obtained the corresponding compressibility curve $\left(\Theta\right.$ vs. $\left.P_{\mathrm{N}}\right)$ it can be fitted by the least squares method to the analytical expression proposed by Secondi [3], which can be expressed in porosity terms as:

$$
\Theta=\Theta_{\infty}+\left(\Theta_{\mathrm{M}}-\Theta_{\infty}\right) \cdot \exp \left(-\left(P_{\mathrm{N}} / a\right)^{b}\right)
$$

where $\Theta$ is the porosity of the powder mass under a compacting pressure $P_{N}$. The parameter $\Theta_{M}$ is known as the tap porosity of the powder [14], being the porosity after a vibration process of the powder inside the die, which will very much depend on morphometric characteristics of the powder as particle shape, size and distribution. The other parameters $\left(\Theta_{\infty}, a\right.$ and $\left.b\right)$ are fitting parameters.

Similarly, the resistivity vs. porosity curve, in the usual situation of having oxide-covered particles, can be fitted by an analytical function of the form [5]:

$$
\rho=c+d\left(1-\Theta / \Theta_{\mathrm{M}}\right)^{-e}
$$

where $c, d$ and $e$ are fitting parameters.

\section{The Method}

Although the curves given by Equations (4) and (5) are determined for a certain powder mass and column section, it does not impede, since they are in analytical form, their use to interpolate any other situation. 


\subsection{Oxide-Covered Powder Particles}

Let us suppose a determined powder mass $(M)$, with bulk density $\gamma_{M}$, inside a die with section $S_{\mathrm{N}}$, and an applied load $F$. Under these conditions, the powder mass will have a porosity $\Theta$, which can be calculated from Equation (4), and will reach a height, according to Equation (1), given by:

$$
H=\frac{M}{S_{\mathrm{N}} \cdot(1-\Theta) \cdot \gamma_{M}}
$$

On the other hand, the effective electrical resistivity of the powder mass is given by Equation (5), evaluated for the previously calculated porosity value. Then, from Equation (2), the electrical resistance of the powder mass results:

$$
R=\rho \frac{H}{S_{\mathrm{N}}}
$$

In case of this value being higher than the maximum allowed value $\left(R_{\mathrm{M}}\right)$, previously known according to the process characteristics and material to be consolidated, the electrical consolidation process will not be possible.

\subsection{Oxide-Free Powder Particles}

In case of having, whichever the process applied (for instance, an activation stage in the electrical consolidation process), oxide-free particles, the metal-oxide-metal contacts will be replaced by metal-metal contacts. Under these conditions, the resistivity of the powder mass will only depend on the metal resistivity and the porosity level. The resistivity of a powder mass, calculated according to Equation (2), with bulk resistivity $\rho_{\mathrm{M}}$, and porosity $\Theta$ determined from Equation (4), can be now fitted by [15]:

$$
\rho=\rho_{\mathrm{M}}\left(1-\Theta / \Theta_{\mathrm{M}}\right)^{-\frac{3}{2}}
$$

which will replace Equation (5) when computing the resistivity.

The resistance value that can now be calculated, in a similar way to the previously described for oxidized particles, represents for the considered porosity the minimum resistance in the powder column when an activation stage has been $100 \%$ effective, which should be lower than the value $R_{\mathrm{M}}$ to have an efficient process.

The intermediate situation, when the activation stage does not completely eliminate the oxide layers, should not be studied with the models described in this paper, or in case of using the models, the correlation coefficients between theoretical and experimental values will result not to be sufficiently good.

\section{Validation}

Commercially pure iron powder (WPL200, from QMP) was used for the validation of the proposed method. This irregular-shape powder (Figure 2) contains as major impurities $0.01 \mathrm{wt}$ \% C, $0.2 \mathrm{Mn}$ and $0.15 \mathrm{O}$. Its absolute density is $7.87 \mathrm{~g} / \mathrm{cm}^{3}$, its apparent density [13] is of $2.65 \mathrm{~g} / \mathrm{cm}^{3}$ and the mean particle size measured by laser diffraction is $78 \mu \mathrm{m}$ (Malvern Mastersizer 2000, Malvern, Worcestershire, UK). The tap porosity was determined to be 0.55 , following the recommendations of the MPIF Standards [14]. 

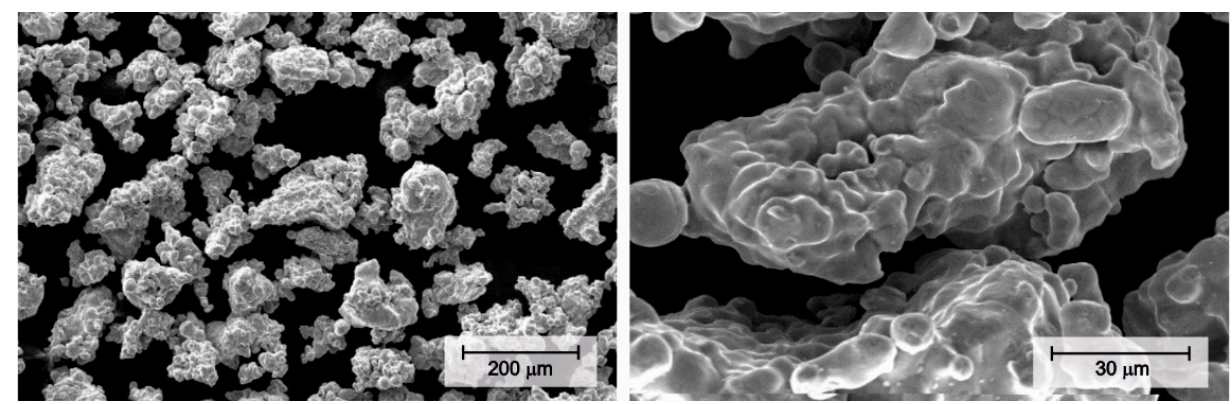

Figure 2. SEM micrographs of the Fe WPL200 powder used in the validation experiments

The validation of the phenomenological model will be tackled in the same way that the model has to be applied to a practical situation. Thus, in an initial experience, the parameters intervening in the model are determined, then, the model can be applied to new situations with different geometries or applied pressure, and with or without activation stage. In the initial experience, and for the compressibility test necessary to determine the curves of compressibility and resistivity, a powder mass of $14 \mathrm{~g}$ inside a $12 \mathrm{~mm}$ diameter die was used. Figure 3a shows the experimental compressibility curve and the fitting by the least squares method to the Secondi expression, Equation (4). This curve and fitting is valid for both the experiences with or without activation stage, because in both situations the powder reaches the desired porosity before the eventual activation stage. Figure $3 \mathrm{~b}$ shows the experimental resistivity vs. porosity curve, and its corresponding fitting through Equation (5).
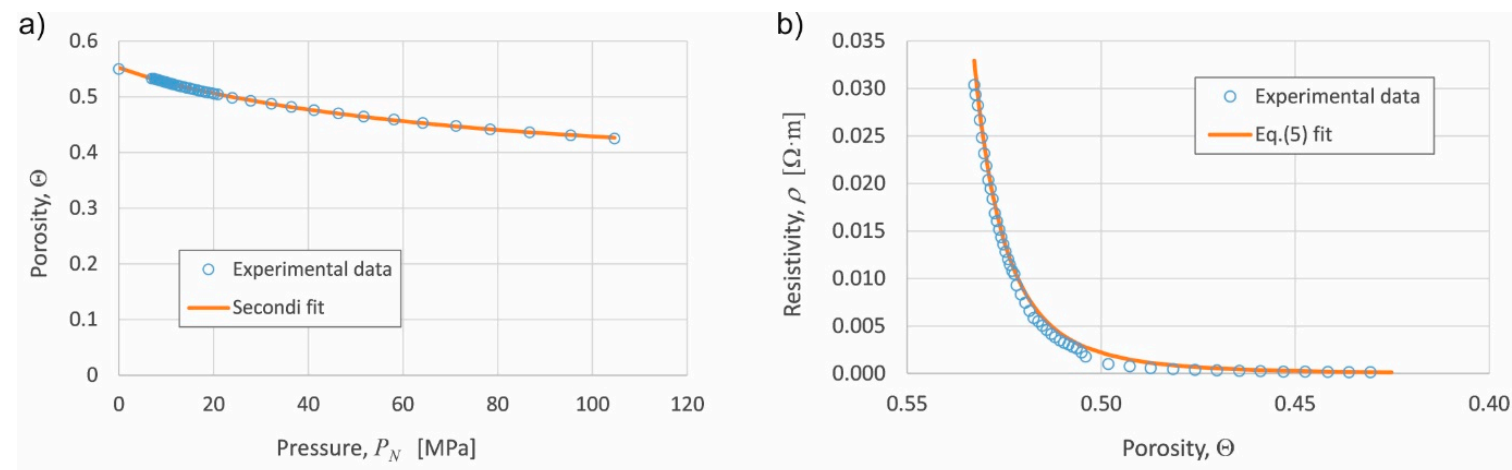

Figure 3. (a) Compressibility curve. (b) Resistivity vs. porosity (please note the decreasing scale) curve.

Table 1 gathers the fitting parameters values in Equations (4) and (5). The determination coefficient $R^{2}$ of Equation (4) fit results 0.9995, and 0.9978 that of Equation (5). As previously detailed, the $\Theta_{\mathrm{M}}$ value was experimentally determined, with a value of 0.55 .

Table 1. Parameters values resulting after fittings the experimental curves with Equations (4) and (5).

\begin{tabular}{ccccccc}
\hline Parameter & $\boldsymbol{a}(\mathbf{M P a})$ & $\boldsymbol{b}$ & $\boldsymbol{\Theta}_{\infty}$ & $\boldsymbol{c}(\boldsymbol{\Omega} \cdot \mathbf{m})$ & $\boldsymbol{d}(\boldsymbol{\Omega} \cdot \mathbf{m})$ & $\boldsymbol{e}$ \\
\hline Value & 68.6460 & 0.9019 & 0.3886 & 0 & $9.8677 \times 10^{-7}$ & 3.4488 \\
\hline
\end{tabular}

Once the initial experience is concluded and the value of the parameters in the model is known, new experiences were carried out. A die with an internal diameter of $8 \mathrm{~mm}$ was chosen. In order to check the goodness of the proposed model, the section of this die was different to the one used to determine the compressibility and resistivity curves. Experiments without activation stage were carried out with three different masses, 6,8 and $12 \mathrm{~g}$, and 4 different pressures $(25,50,75$ and $100 \mathrm{MPa})$, gathering a total of 12 combinations. In all the experiments, the electrical resistance of the powder 
column subjected to compression was determined. The measured values and those predicted by using the described process (using Equations (4) and (5) with the parameters shown in Table 1), as well as the resulting relative error, are shown in Table 2 . Values differed by less than a $2 \%$, with a mean relative error of $0.83 \%$.

Table 2. Measured and modeled resistance values for experiments without activation stage.

\begin{tabular}{cccccc}
\hline Mass & Property & $\mathbf{2 5} \mathbf{~ M P a}$ & $\mathbf{5 0} \mathbf{~ M P a}$ & $\mathbf{7 5} \mathbf{~} \mathbf{P a}$ & $\mathbf{1 0 0} \mathbf{~} \mathbf{P a}$ \\
\hline \multirow{3}{*}{$\mathbf{6} \mathbf{g}$} & $R$ (measured) $(\Omega)$ & 1.5958 & 0.3040 & 0.1343 & 0.0817 \\
& $R$ (predicted) $(\Omega)$ & 1.6090 & 0.3007 & 0.1328 & 0.0820 \\
& Relative Error $(\%)$ & 0.83 & 1.07 & 1.13 & 0.46 \\
\hline \multirow{3}{*}{$\mathbf{g}$} & $R$ (measured) $(\Omega)$ & 2.1667 & 0.4018 & 0.1784 & 0.1089 \\
& $R$ (predicted) $(\Omega)$ & 2.1454 & 0.4010 & 0.1771 & 0.1094 \\
& Relative Error $(\%)$ & 0.98 & 0.19 & 0.73 & 0.44 \\
\hline \multirow{2}{*}{$\mathbf{1 2} \mathbf{g}$} & $R$ (measured) $(\Omega)$ & 3.2825 & 0.5950 & 0.2655 & 0.1624 \\
& $R$ (predicted) $(\Omega)$ & 3.2181 & 0.6015 & 0.2656 & 0.1641 \\
& Relative Error $(\%)$ & 1.96 & 1.09 & 0.02 & 1.04 \\
\hline
\end{tabular}

In order to validate the activation stage effect via the oxide-free particles model (Equation (8)), an electrical discharged was applied to the powder column after reaching the desired porosity and before each resistance measurement, under the same conditions aforementioned. The electrical discharge came from an autotransformer capable of providing a voltage from 0 to $220 \mathrm{~V}$ and a maximum intensity of $10 \mathrm{~A}$, protected by a magnetothermic. The device slowly increased voltage until the magnetothermic opened the circuit. Then the resistance of the powder column was measured, and also calculated according to Equation (8) by considering the resistivity of the bulk material $\rho_{\mathrm{M}}=10.1 \times 10^{-8} \Omega \cdot \mathrm{m}[16]$ and the porosity calculated from Equation (4). Resulting measured and predicted values are shown in Table 3. Now, the maximum relative error in the analyzed data is a $9.5 \%$, with seven of the experiences with an error higher than the $5 \%$, and with a mean relative error of $4.9 \%$.

Table 3. Measured and modeled resistance values for experiments with a previous activation stage.

\begin{tabular}{cccccc}
\hline Mass & Property & $\mathbf{2 5} \mathbf{~ M P a}$ & $\mathbf{5 0 ~ M P a}$ & $\mathbf{7 5} \mathbf{~ M P a}$ & $\mathbf{1 0 0} \mathbf{~ M P a}$ \\
\hline \multirow{3}{*}{$\mathbf{6} \mathbf{g}$} & $R$ (measured) $(\Omega)$ & $1.7479 \times 10^{-3}$ & $8.8793 \times 10^{-4}$ & $5.4458 \times 10^{-4}$ & $4.8906 \times 10^{-4}$ \\
& $R$ (predicted) $(\Omega)$ & $1.8753 \times 10^{-3}$ & $8.7124 \times 10^{-4}$ & $5.9619 \times 10^{-4}$ & $4.7581 \times 10^{-4}$ \\
& Relative Error $(\%)$ & 7.29 & 1.88 & 9.48 & 2.71 \\
\hline \multirow{2}{*}{$\mathbf{8} \mathbf{g}$} & $R$ (measured) $(\Omega)$ & $2.3365 \times 10^{-3}$ & $1.0989 \times 10^{-3}$ & $7.3329 \times 10^{-4}$ & $6.2951 \times 10^{-4}$ \\
& $R$ (predicted) $(\Omega)$ & $2.5005 \times 10^{-3}$ & $1.1617 \times 10^{-3}$ & $7.9492 \times 10^{-4}$ & $6.3442 \times 10^{-4}$ \\
& Relative Error $(\%)$ & 7.02 & 5.71 & 8.40 & 0.78 \\
\hline \multirow{2}{*}{$\mathbf{1 2} \mathbf{g}$} & $R$ (measured) $(\Omega)$ & $3.8132 \times 10^{-3}$ & $1.6179 \times 10^{-3}$ & $1.2652 \times 10^{-3}$ & $9.5094 \times 10^{-4}$ \\
& $R$ (predicted) $(\Omega)$ & $3.7507 \times 10^{-3}$ & $1.7425 \times 10^{-3}$ & $1.1924 \times 10^{-3}$ & $9.5163 \times 10^{-4}$ \\
& Relative Error $(\%)$ & 1.64 & 7.70 & 5.75 & 0.07 \\
\hline
\end{tabular}

It is worth noting that a quick analysis from the previous data, with the resistance values shown in Table 2, without activation stage, is two or three orders of magnitude higher than those in Table 3. The difference has to be attributed to the presence of oxide layer in the experiences without activation stage, although partially descaled by the pressure effect, making the powders to partially decrease their resistivity. On the other hand, as expected, the activation stage breaks the oxide layers decreasing the resistivity, although the higher differences between the experimental and predicted values in the case of activation stage can be indicative of a non-total oxide elimination. Certainly, the activation process also has an erratic component because of the avalanche processes in electrically favorable paths, making the process not to be uniform and deviating the process of the proposed model. Nevertheless, the experiences show the efficiency of the activation stage in order to reduce the value of $R_{\mathrm{M}}$, which is 
translated in the practical possibility of undertaking the electrical consolidation of more resistive powders or higher powder columns.

Despite the aforementioned relative errors, and according to the measured and predicted values in Tables 2 and 3, it can be concluded that the model can be considered to be satisfactorily validated. In this way, results show that it is possible to estimate the electrical resistance of a powder mass under compression (with or without activation stage), and by comparing with the $R_{\mathrm{M}}$ value, to determine whether the planed consolidation experience will or not be feasible, with the consequent save in the number of experiences.

\section{Conclusions}

A valid method to predict the electrical resistance of a powder mass under compression, before and after being subjected to a process of electrical activation (which provokes the dielectric breakdown of the oxide layers), has been proposed. The method requires the experimental determination of both the compressibility and the electrical resistivity vs. porosity curves, and the fitting of the obtained data to the analytical expressions here presented. The results obtained in the validation with simple and direct experiences, confirm the goodness of the proposed method.

Acknowledgments: Financial support of the Ministerio de Economía y Competitividad (Spain) and Feder (EU) through the research projects DPI2015-69550-C2-1-P and DPI2015-69550-C2-2-P are gratefully acknowledged. The authors also wish to thank the technicians J. Pinto, M. Madrid and M. Sánchez (University of Seville, Spain) for experimental assistance.

Author Contributions: Juan Manuel Montes and Francisco Gómez Cuevas designed the experiences, analyzed results and wrote the manuscript, Fátima Ternero, Raquel Astacio and Eduardo Sánchez Caballero obtained the experimental results, Jesús Cintas worked on the characterization of the starting material.

Conflicts of Interest: The authors declare no conflict of interest.

\section{References}

1. Grasso, S.; Sakka, Y.; Maizza, G. Electric current activated/assisted sintering (ECAS): A review of patents 1906-2008. Sci. Technol. Adv. Mater. 2009. [CrossRef] [PubMed]

2. Montes, J.M.; Cuevas, F.G.; Cintas, J. Effective area in powder compacts under uniaxial compression. Mater. Sci. Eng. 2005, 395, 208-213. [CrossRef]

3. Secondi, J. Modeling powder compaction-From a pressure-density law to continuum mechanics. Powder Metall. 2002, 45, 213-217. [CrossRef]

4. Evans, U.R. The Corrosion and Oxidation of Metals: First Supplementary Volume; Edward Arnold: London, UK, 1968.

5. Garino, T.J. Electrical behavior of oxidized metal powders during and after compaction. J. Mater. Res. 2002, 17, 2691-2697. [CrossRef]

6. Montes, J.M.; Cuevas, F.G.; Cintas, J.; Urban, P. Electrical conductivity of metal powders under pressure. Appl. Phys. A Mater. Sci. Process. 2011, 105, 935-947. [CrossRef]

7. Montes, J.M.; Cuevas, F.G.; Cintas, J.; Gallardo, J.M. Electrical conductivity of metal powder aggregates and sintered compacts. J. Mater. Sci. 2016, 51, 822-835. [CrossRef]

8. Kingery, W.D.; Bowen, H.K.; Uhlmann, D.R. Introduction to Ceramics, 2nd ed.; John Wiley \& Sons: New York, NY, USA, 1976.

9. Tsuda, N.; Nasu, K.; Fujimori, A.; Siratori, K. Electronic Conduction in Oxides, 2nd ed.; Springer: New York, NY, USA, 2000.

10. Akl, A.A. Microstructure and electrical properties of iron oxide thin films deposited by spray pyrolysis. Appl. Surf. Sci. 2004, 221, 319-329. [CrossRef]

11. Wakefield, G.; Dobson, P.J.; Foo, Y.Y.; Loni, A.; Simons, A.; Hutchison, J.L. The fabrication and characterization of nickel oxide films and their application as contacts to polymer/porous silicon electroluminescent devices. Semicond. Sci. Technol. 1997, 12, 1304-1309. [CrossRef]

12. Jones, L.D.; Chin, A.F. Electronic Instruments and Measurements, 2nd ed.; Prentice-Hall: Upper Saddle River, NJ, USA, 1991. 
13. M. Standard 04. Determination of Apparent Density of Free-Flowing Metal Powders Using the Hall Apparatus. In Standard Test Methods for Metal Powders and Powder Metallurgy Products; Metal Powder Industries Federation (MPIF): Princeton, NJ, USA, 2002.

14. M. Standard 46. Determination of Tap Density of Metal Powders. In Standard Test Methods for Metal Powders and Powder Metallurgy Products; Metal Powder Industries Federation (MPIF): Princeton, NJ, USA, 2002.

15. Montes, J.M.; Cuevas, F.G.; Cintas, J. Porosity effect on the electrical conductivity of sintered powder compacts. Appl. Phys. A Mater. Sci. Process. 2008, 92, 375-380. [CrossRef]

16. Gale, W.F.; Totemeier, T.C. Smithells Metals Reference Book, 8th ed.; Elsevier: Toronto, ON, Canada, 2003.

(C) 2017 by the authors. Licensee MDPI, Basel, Switzerland. This article is an open access article distributed under the terms and conditions of the Creative Commons Attribution (CC BY) license (http:/ / creativecommons.org/licenses/by/4.0/). 